\title{
Relation between dispersion lines and conductance of telescoped armchair double-wall nanotubes analyzed using perturbation formulas and first-principles calculations
}

\author{
Ryo Tamura \\ Faculty of Engineering, Shizuoka University, 3-5-1 Johoku, Hamamatsu 432-8561, Japan
}

\begin{abstract}
The Landauer's formula conductance of the telescoped armchair nanotubes is calculated with the Hamiltonian defined by first-principles calculations (SIESTA code). Herein, partially extracting the inner tube from the outer tube is called 'telescoping'. It shows a rapid oscillation superposed on a slow oscillation as a function of discrete overlap length $\left(L-\frac{1}{2}\right) a$ with an integer variable $L$ and the lattice constant a. Considering the interlayer Hamiltonian as a perturbation, we obtain the approximate formula of the amplitude of the slow oscillation as $|A|^{2} /\left(|A|^{2}+\varepsilon^{2}\right)$ where $A$ is the effective interlayer interaction and $\varepsilon$ is the band split without interlayer interaction. The approximate formula is related to the Thouless number of the dispersion lines.
\end{abstract}

\section{INTRODUCTION}

Single-wall nanotubes (SWNT) show various useful characteristics as nanoelectronic devices. ${ }^{1,2}$ They can be either metallic or semiconducting, depending on their chirality. ${ }^{3}$ Although a semiconducting SWNT can act as a transistor by itself, ${ }^{4}$ we can increase NT device functionality by assembling SWNTs. For example, a junction between a metallic SWNT and a semiconducting SWNT can work as a Schottky diode. ${ }^{5,6}$ The SWNTs can be connected by covalent bonds ${ }^{5,7}$ and interlayer interactions. ${ }^{6,8}$ Double-wall nanotubes (DWNTs), multiwall nanotubes (MWNTs), and nanotube bundles are all examples of SWNTs that can be naturally assembled through interlayer interactions. Processing these naturally assembled SWNTs is one way of constructing higher-order structures for NT devices. One prototype higher-order structure is the telescoped double wall nanotube (TDWNT), in which the inner tube is partially extracted from a DWNT, as shown in Fig. 1. Since the interlayer force is relatively small, the inner tube can be slid and rotated with respect to the outer tube by attached piezoelectric probes with a negligible change in intralayer bonding. ${ }^{9,10}$ In this way, we can realize various interlayer configurations in the TDWNT, unlike the case for bulk graphite. When a bias voltage is applied between the attached probes, the resulting current is significantly influenced by the interlayer configuration because a carrier from one probe must travel an interlayer path to reach the other probe. This sensitivity can be used in nanomechanical switches and nanodisplacement sensors. In addition, TDWNTs enable us to measure the interlayer conductance more directly than un-telescoped DWNTs. ${ }^{11}$

Among the reported theo-

retical work on TDWNTs, ${ }^{12-15}$, there are controversial results regarding the conductance of a TDWNT composed of $(10,10)$ and $(5,5)$ armchair nanotubes. Compared to a quantum conductance $G_{0}=2 e^{2} / h$, the maximum conductance was found to be $2 G_{0}$ based on tight binding (TB) model calculations, but only $G_{0}$ based on first-principles calculations. ${ }^{14,15}$ Landauer's formula was used in these calculations, so the conductance in units of $G_{0}$ equals the sum of the transmission rates. In Ref. ${ }^{15}$, the author derived approximate formulas to clarify the direct relationship between the transmission rate and the interlayer Hamiltonian. The relation between the transmission rates and the dispersion lines was used in this derivation. The energy $E$, Hamiltonian $H$, and overlap matrix $B$ were common to the exact calculations and the approximate formulas. The approximate formulas indicated that the disagreement comes from differences in the interlayer Hamiltonian. The interlayer Hamiltonian is also important in multilayer graphenes ${ }^{16}$ with regard to, e.g., transmission through a boundary between monolayer and bilayer graphene. ${ }^{17}$ The TB Hamiltonian was used in Ref. ${ }^{15}$, but is insufficient for the interpretation of the first-principles calculations. Thus, both the exact method and the approximate formulas were adapted to a first-principles calculation code with an atomic orbital (AO) basis. The effectiveness of the approximate formulas was judged from their agreement with exact calculations.

\section{INITIAL GEOMETRIC STRUCTURE AND SITE INDEX}

Geometric optimization was performed with the initial structure defined as follows. In the initial structure, cylindrical coordinates $(r, \theta, z)=\left(r^{\mu}, \theta_{l, m}^{\mu}, z_{j, m}^{\mu}\right)$ of the carbon atoms in the tube $\mu=O$ (outer tube) and $\mu=I$ (inner tube) are represented by

$$
\begin{gathered}
\theta_{l, m}^{\mu}=\frac{\pi}{n_{\mu}}\left(l+\frac{-5-(-1)^{l+m}}{6}\right) \\
z_{j, m}^{\mu}=0.5 a(2 j+m) .
\end{gathered}
$$

The notation of Eqs. (1) and (2) is the same as Ref. ${ }^{15}$ except that the integer index $j$ in Ref. ${ }^{15}$ is replaced by $2 j+m$ here. The radius of the tube $\mu$ is denoted as $r^{\mu}=\sqrt{3} a n_{\mu} /(2 \pi)$, with integers $n_{O}=10, n_{I}=5$ and the lattice constant $a=\sqrt{3} \times 0.142 \mathrm{~nm}$. The unit cells are 
numbered $j$ along the tube axis, while the atoms in each unit cell are numbered $m=-1,0$ and $l=1,2, \cdots, 2 n_{\mu}$. As the DWNT considered here has no mirror plane parallel to the tube axis, symmetric and anti-symmetric states are hybridized by the interlayer interaction. We use $\mu, l, j, m$ as site indexes in the following. We discuss three structures: an un-telescoped DWNT with five unit cells, a TDWNT, and an un-telescoped DWNT with infinite length. These are denoted as [fi], [td], and [in]. Although Eqs. (1) and (2) are common among [fi], [td], and [in], the range of $j$ at which the corresponding atoms exist is different, as

$$
\begin{aligned}
1 \leq j \leq 5 \quad \text { in }[\mathrm{fi}] \\
1 \leq j<\infty \quad \text { in tube } O \text { of }[\mathrm{td}] \\
-\infty \leq j \leq L \text { in tube } I \text { of }[\mathrm{td}]
\end{aligned}
$$

and

$$
-\infty<j<\infty \quad \text { in [in]. }
$$

where $L$ denotes the number of unit cells in the DWNT of [td]. In the initial structure of [fi], the direction of the $\mathrm{C}-\mathrm{H}$ bond is the same as the corresponding $\mathrm{C}-\mathrm{C}$ bond and the $\mathrm{C}-\mathrm{H}$ bond length is $0.11 \mathrm{~nm}$.

\section{BUILDING BLOCK PROCEDURE}

The SIESTA code is used as the first-principles calculation of structure [fi]..$^{1{ }^{-20}}$ The geometry is optimized by the conjugate gradients method with the convergence criteria $0.04 \mathrm{eV} /$ Ang. The optimized and initial structures are superimposed on Fig. 2. We can see that the initial structure is almost maintained after the optimization. The minimum, average and maximum bond lengths are $0.1175,0.1177,0.1181 \mathrm{~nm}$ for $\mathrm{C}-\mathrm{H}$ and $0.1413,0.1451$, 0.1482 for C-C. The output data after the geometrical optimization are used. Since the obtained Hamiltonian matrix has an atomic orbital (AO) basis, it can be divided into block matrices based on the unit cells. The one-electron wave function $\psi$ is represented by the linear combination of $\mathrm{AOs}\{\phi\}$, as

$$
\psi=\sum_{\mu=I, O} \sum_{j} \sum_{l=1}^{2 n_{\mu}} \sum_{m=-1}^{0} \sum_{\alpha} d_{l, m, \alpha, j}^{\mu} \phi_{l, m, \alpha, j}^{\mu} .
$$

In Eq. (6), four single zeta $\operatorname{AOs}\left(\alpha=2 \mathrm{~s}, 2 \mathrm{p}_{x}, 2 \mathrm{p}_{y}, 2 \mathrm{p}_{z}\right)$ are considered per atom.

The secular equations for structures [in] and [td] are represented by

$$
\sum_{\mu^{\prime}=I, O} \sum_{j^{\prime}} H_{j, j^{\prime}}^{\mu, \mu^{\prime}} \vec{d}_{j^{\prime}}^{\mu^{\prime}}=E \sum_{\mu^{\prime}=I, O} \sum_{j^{\prime}} B_{j, j^{\prime}}^{\mu, \mu^{\prime}} \vec{d}_{j^{\prime}}^{\mu^{\prime}}
$$

where $H_{j, j^{\prime}}^{\mu, \mu^{\prime}}$ and $B_{j, j^{\prime}}^{\mu, \mu^{\prime}}$ are the block matrices of $H$ and $B$, respectively. The vector $\vec{d}_{j}^{\mu}$ is composed of $d_{l, m, \alpha, j}^{\mu}$ in
Eq. (6). The ranges of $j^{\prime}$ and $j$ in Eq. (7) are shown in Eqs. (4) and (5). From the obtained $H_{j, j^{\prime}}^{\mu, \mu^{\prime},[\mathrm{fi}]}$ and $B_{j, j^{\prime}}^{\mu, \mu^{\prime},[\mathrm{fi}]}$, the block matrices of structure [in] are defined as

$$
H_{\eta, \eta+\Delta j}^{\mu, \mu^{\prime}}={ }^{t} H_{\eta+\Delta j, \eta}^{\mu^{\prime}, \mu} \equiv H_{3,3+\Delta j}^{\mu, \mu^{\prime},[\mathrm{fi}]}
$$

for $\left(\mu, \mu^{\prime}\right)=(I, I),(O, I),(I, O)$,

$$
H_{\eta, \eta+\Delta j}^{O, O}={ }^{t} H_{\eta+\Delta j, \eta}^{O, O} \equiv H_{3,3+\Delta j}^{O, O,[\mathrm{fi}]}+\Delta \varepsilon B_{3,3+\Delta j}^{O, O,[\mathrm{fi}]}
$$

and

$$
B_{\eta, \eta+\Delta j}^{\mu, \mu^{\prime}}={ }^{t} B_{\eta+\Delta j, \eta}^{\mu^{\prime}, \mu} \equiv B_{3,3+\Delta j}^{\mu, \mu^{\prime},[\mathrm{fi}]}
$$

where $\eta$ is an arbitrary integer and $\Delta j=0,1,2$. When $|\Delta j|>2, B_{\eta, \eta+\Delta j}^{\mu^{\prime}, \mu} \equiv 0$ and $H_{\eta, \eta+\Delta j}^{\mu^{\prime}, \mu,[\xi]} \equiv 0$. Here we select block matrices $H^{[\mathrm{i}]}, B^{[\mathrm{fi}]}$ for which the boundary effect is relatively small and build them into the $H$ and $B$ matrixes of structure [in]. Once we obtain $H^{[\mathrm{fi}]}$ and $B^{[\mathrm{fi}]}$ from the SIESTA, there is no adjustable parameter except for $\Delta \varepsilon$. The purpose of introducing the parameter $\Delta \varepsilon$ will be explained in Sec. IV. The $H$ and $B$ matrices of structure [td] are defined also by the building block procedure as will be shown in Sec.VB.

\section{DISPERSION RELATION}

In order to discuss the dispersion relation of structure [in], it is useful to define

$$
X^{D, D} \equiv\left(\begin{array}{ll}
X^{I, I} & X^{I, O} \\
X^{O, I} & X^{O, O}
\end{array}\right)
$$

and

$$
\widetilde{X}^{\mu^{\prime}, \mu}(k) \equiv \sum_{j=-M}^{M} X_{0, j}^{\mu^{\prime}, \mu} e^{i k a j}
$$

where $X=H, B$. The dispersion relation $E_{\tau}^{\mu}(k)$ and the wave function per unit cell $\vec{g}_{\tau}^{\mu}(k)$ can be obtained by

$$
\widetilde{H}^{\mu, \mu}(k) \vec{g}_{\tau}^{\mu}(k)=E_{\tau}^{\mu}(k) \widetilde{B}^{\mu, \mu}(k) \vec{g}_{\tau}^{\mu}(k)
$$

with the band index $\tau$. According to Eqs. (11), (12) and $(13), E_{\tau}^{D}(k)$ corresponds to a DWNT with interlayer interaction, while $E_{\tau}^{I}(k)$ and $E_{\tau}^{O}(k)$ correspond to isolated SWNTs.

The dispersion lines near the neutral Fermi level $E_{F}^{(0)}$ are denoted by $E_{ \pm 1}^{D}, E_{ \pm 2}^{D}, E_{ \pm 3}^{D}, E_{ \pm 4}^{D}$ for a DWNT with interlayer interaction, and $E_{ \pm 1}^{O}, E_{ \pm 1}^{I} E_{ \pm 2}^{O}$ and $E_{ \pm 2}^{I}$ for isolated tubes $O$ and $I$. Figure 3 shows the dispersion lines numbered based on the index $\tau$ under the conditions $E_{|\tau|}^{\mu}\left((-1)^{\tau} \frac{2 \pi}{3 a}\right)=E_{-|\tau|}^{\mu}\left(-(-1)^{\tau} \frac{2 \pi}{3 a}\right) \simeq E_{F}^{(0)}$ and $\tau \frac{d E_{\tau}^{\mu}}{d k}>$ 0 . For the isolated tubes $\mu=I$ and $\mu=O, E_{ \pm 1}^{\mu}$ and $E_{ \pm 2}^{\mu}$ correspond to the symmetric and anti-symmetric states, 
respectively, with respect to the mirror plane parallel to the tube axis. For a DWNT with interlayer interaction, however, the mirror symmetry is broken and the band splitting is increased as $E_{3}^{D}-E_{1}^{D}>\left|E_{1}^{O}-E_{1}^{I}\right|$ and $E_{4}^{D}-$ $E_{2}^{D}>\left|E_{2}^{O}-E_{2}^{I}\right|$.

Figure 4 shows the dispersion relation of the isolated SWNTs for $M=1$ (open circles) and $M=2$ (closed diamonds). The shift between $M=1$ and $M=2$ is about $0.002 \sim 0.003 \mathrm{Ry}$ for the $E_{-1}$ band. In order to include this energy difference, the integer $M$ in Eq. (12) is set to 2 hereafter. Squares and circles in Fig. 5 show the intralayer and interlayer effective Hamiltonian elements $h\left(\vec{r}, \vec{r}^{\prime}\right)$, respectively, for the atomic distance $0.3 \mathrm{~nm}<\left|\vec{r}-\vec{r}^{\prime}\right|<0.45 \mathrm{~nm}$. The effective Hamiltonian $h\left(\vec{r}, \vec{r}^{\prime}\right)$ is defined by

$$
h \equiv\left|\frac{\sum_{\alpha, \alpha^{\prime}} g_{\vec{r}, \alpha}^{*} H g_{\vec{r}^{\prime}, \alpha^{\prime}}}{\sqrt{\sum_{\alpha, \alpha^{\prime}} g_{\vec{r}, \alpha}^{*} B g_{\vec{r}, \alpha^{\prime}}} \sqrt{\sum_{\alpha, \alpha^{\prime}} g_{\vec{r}^{\prime}, \alpha}^{*} B g_{\vec{r}^{\prime}, \alpha^{\prime}}}}\right|
$$

where $g_{\vec{r}, \alpha}$ denotes the component of $\vec{g}_{-1}^{I}, \vec{g}_{-1}^{O}$ for $k=0.66 \pi / a$, atomic position $\vec{r}$ and $\mathrm{AO}$ type $\alpha=2 \mathrm{~s}, 2 \mathrm{p}_{x}, 2 \mathrm{p}_{x}, 2 \mathrm{p}_{z}$. The spatial range of the effective Hamiltonian is considerably longer compared to the TB Hamiltonian used in Ref. ${ }^{15}$. Crosses represent the intralayer elements between the second nearest neighbor unit cells. They cause the energy difference between $M=1$ and $M=2$ in Fig. 4 .

According to Ref. ${ }^{15}$, we define the intrinsic shift as

$$
\varepsilon_{\tau} \equiv E_{\tau}^{O}(k)-E_{\tau}^{I}(k)
$$

Figure 4 shows a reasonable value of $\frac{d E^{\mu}}{d k}$. However, $\left|\varepsilon_{\tau}(k)\right|$ is considerably larger than other first-principles calculation results, probably because of the finite size effect of structure [fi] used in the first step of the building block procedure. The parameter $\Delta \varepsilon$ in Eq. (8) changes $\varepsilon_{\tau}$ as

$$
\varepsilon_{\tau}=\varepsilon_{\tau}^{(0)}+\Delta \varepsilon
$$

where $\varepsilon_{\tau}^{(0)}$ denotes the intrinsic shift in Fig. 4. As $\Delta \varepsilon$ is common to the exact and approximate methods, it is not a parameter that can be adjusted to produce agreement between the results obtained by the two methods. Figure 4 shows that $\varepsilon_{-1}^{(0)}=-0.022 \mathrm{Ry}$ and $\varepsilon_{2}^{(0)}=-0.028 \mathrm{Ry}$ at $k=0.65 \pi / a$.

When $\mu=D$, Eq. (13) can be transformed to

$$
{ }^{t} \vec{g}_{\tau^{\prime}}^{D}(k)^{*}\left[\widetilde{H}^{D, D}(k)-E_{\tau^{\prime}}^{D}(k) \widetilde{B}^{D, D}(k)\right] \vec{g}_{\tau^{\prime}}^{D}(k)=0
$$

In the following, we consider the wavenumber $k$ as a function of the energy $E$. The wavenumbers of the isolate tubes $I$ and $O$ are denoted by $k^{I}(E)$ and $k^{O}(E)$, respectively. Neglecting mixing between different $\tau$ values, the lowest order approximation is represented by

$$
{ }^{t} \vec{g}_{\tau^{\prime}}^{D}(k) \simeq\left(y_{I}{ }^{t} \vec{g}_{\tau}^{I}\left(k^{I}\right), y_{O}{ }^{t} \vec{g}_{\tau}^{O}\left(k^{O}\right)\right)
$$

where $\tau^{\prime}=\tau, \tau+2 \frac{\tau}{\tau \mid}$. Here the three different wavenumbers have the common energy as $E=E^{D}(k)=E^{I}\left(k^{I}\right)=$ $E^{O}\left(k^{O}\right)$ and $k \neq k^{I} \neq k^{O}$.

Using Eq. (18) and

$$
\tilde{X}^{\mu^{\prime}, \mu}(k) \simeq \tilde{X}^{\mu^{\prime}, \mu}\left(k^{\mu}\right)+\left.\left(k-k^{\mu}\right) \frac{d}{d k} \tilde{X}^{\mu^{\prime}, \mu}\right|_{k=k^{\mu}},
$$

Eq. (17) can be approximated by

$$
\operatorname{Re}\left[\left(A_{\tau}+C_{\tau}\right) y_{O}^{*} y_{I}\right]+\sum_{\mu=I, O} \frac{k-k_{\tau}^{\mu}}{\sqrt{b_{\tau}^{I} b_{\tau}^{O}}} b_{\tau}^{\mu} \frac{d E_{\tau}^{\mu}}{d k}\left|y_{\mu}\right|^{2} \simeq 0
$$

where

$$
\begin{gathered}
b_{\tau}^{\mu} \equiv{ }^{t} \vec{g}_{\tau}^{\mu}\left(k^{\mu}\right)^{*} \widetilde{B}^{\mu, \mu}\left(k^{\mu}\right) \vec{g}_{\tau}^{\mu}\left(k^{\mu}\right) \\
b_{\tau}^{\mu} \frac{d E_{\tau}^{\mu}}{d k}=\left.\left({ }^{t} \vec{g}_{\tau}^{\mu *} \frac{d \widetilde{P}^{\mu, \mu}(k)}{d k} \vec{g}_{\tau}^{\mu}\right)\right|_{k=k^{\mu}} \\
A_{\tau} \equiv \frac{2^{t} \vec{g}_{\tau}^{O}\left(k^{O}\right)^{*} \widetilde{P}^{O, I}\left(k^{I}\right) \vec{g}_{\tau}^{I}\left(k^{I}\right)}{\sqrt{b_{\tau}^{O} b_{\tau}^{I}}} \\
\left.\frac{2\left(k-k^{I}\right)}{\sqrt{b_{\tau}^{O} b_{\tau}^{I}}}{ }^{t} \vec{g}_{\tau}^{O *}\left(k_{\tau}^{O}\right)\left(\frac{d \widetilde{P}^{O, I}}{d k} \vec{g}_{\tau}^{I}\right)\right|_{k=k^{I}} \\
\widetilde{P}^{\mu, \mu^{\prime}}(k) \equiv \widetilde{H}^{\mu, \mu^{\prime}}(k)-E \widetilde{B}^{\mu, \mu^{\prime}}(k) \\
\frac{d \widetilde{P}^{\mu, \mu^{\prime}}}{d k} \equiv \frac{d \widetilde{H}^{\mu, \mu^{\prime}}}{d k}-E \frac{d \widetilde{B}^{\mu, \mu^{\prime}}}{d k}
\end{gathered}
$$

Here Eq. (22) will be proved in Appendix B.

From Eq. $(20), k_{\tau^{\prime}}^{D}$ can be approximated by

$$
k_{\tau^{\prime}}^{D, A}(E)=\frac{1}{2}\left[k_{\tau}^{I}(E)+k_{\tau}^{O}(E) \pm \Delta k_{\tau}^{D, A}\right]
$$

where

$$
\begin{gathered}
\Delta k_{\tau}^{D, A} \equiv\left|\Delta \widetilde{k}_{\tau}\right| \sqrt{1+x_{\tau}} \\
\Delta \widetilde{k}_{\tau} \equiv k_{\tau}^{O}(E)-k_{\tau}^{I}(E)
\end{gathered}
$$

and $x_{\tau}$ is a dimensionless parameter defined by

$$
x_{\tau} \equiv\left|A_{\tau}\right|^{2}\left|\frac{d k^{I}}{d E} \frac{d k^{O}}{d E}\right|(\Delta \widetilde{k})^{-2} .
$$

The band split along the $k$ axis without interlayer interaction is denoted by Eq.(29), while that with interlayer interaction

$$
\Delta k_{\tau}^{D} \equiv\left|k_{\tau+2}^{D} \frac{\tau}{|\tau|}(E)-k_{\tau}^{D}(E)\right|
$$

is approximated by Eq.(28). Since $|C| \ll|A|$, the effect of Eq.(24) is neglected here. Note that we define $\vec{g}_{\tau}^{O}, \vec{g}_{\tau}^{I}$, $b_{\tau}^{O} b_{\tau}^{I}, k_{\tau}^{O}$ and $k_{\tau}^{I}$ without the interlayer matrixes $H^{O, I}$ and $B^{O, I}$. 


\section{TRANSMISSION RATE}

In the following, $T_{\tau, \tau^{\prime}}$ denotes the transmission rate from the $\tau^{\prime}(=1,2)$ channel of tube $I$ to the $\tau(=1,2)$ channel of tube $O$. Here, the notation of $\tau$ is common with that of Sec. IV, i.e., $\tau=1$ and $\tau=2$ are symmetric and anti-symmetric channels, respectively.

Since the interlayer Hamiltonian breaks the mirror symmetry, the inter-channel transmission rates $T_{1,2}$ and $T_{2,1}$ are not zero. Nevertheless, the large difference between $k_{1} \simeq-2 \pi /(3 a)$ and $k_{2} \simeq 2 \pi /(3 a)$ suppresses $T_{1,2}$ and $T_{2,1}$. In the approximate formulas, we consider only the intra-channel transmission rates $T_{1,1}$ and $T_{2,2}$.

\section{A. Approximate formula}

In order to clarify the relation of Eq. (23) to the effective interlayer interaction of Ref. ${ }^{15}$, we transform Eq. (23) into

$$
A=\frac{2 \int \chi^{O}(1)^{*}(\widehat{H}-E) \Psi^{I}(k) d^{3} \vec{r}}{\sqrt{\int \chi^{O}(1)^{*} \Psi^{O}(k) d^{3} \vec{r}} \sqrt{\int \chi^{I}(1)^{*} \Psi^{I}(k) d^{3} \vec{r}}}
$$

where $\widehat{H}$ is the Hamiltonian operator,

$$
\begin{gathered}
\Psi^{\mu}(k) \equiv \sum_{j=-\infty}^{\infty} e^{i k a j} \chi^{\mu}(j) \\
\chi^{\mu}(j) \equiv \sum_{l=1}^{2 n_{\mu}} \sum_{m=-1}^{0} \sum_{\alpha} g_{l, m, \alpha, j}^{\mu}(k) \phi_{l, m, \alpha, j}^{\mu}
\end{gathered}
$$

and $g_{l, m, \alpha, j}^{\mu}(k)$ denotes the component of $\vec{g}^{\mu}(k)$. Equation (32) clearly indicates that $\frac{A}{2}$ equals the matrix element of the perturbation $H^{O, I}-E B^{O, I}$ between the unperturbed states $\Psi^{O}$ and $\Psi^{I}$ per unit cell. When $\mu=O, I$, Eq. (33) is the eigenfunction of the intralayer Hamiltonian $H^{\mu, \mu}$ and bears no relation to the interlayer Hamiltonian $H^{O, I}$. Since $A$ of Ref. ${ }^{15}$ can also be represented by Eq. (32), the physical meaning of $A$ is common to the present paper and Ref. ${ }^{15}$. There is, however, another possible definition of $A$ where $\widetilde{P}^{O, I}=\widetilde{H}^{O, I}-E \widetilde{B}^{O, I}$ is replaced by $\widetilde{H}^{O, I}$. The two definitions cause no difference when the interlayer overlap matrix $B^{O, I}$ is absent. Though the effect of $E \widetilde{B}^{O, I}$ is also investigated in Sec.VI, the definition (23) is chosen here.

The approximate formula of Ref. ${ }^{15}$ is represented by

$$
T_{\tau, \tau}^{\prime}=\frac{\left|A_{\tau}\right|^{2}}{\varepsilon_{\tau}^{2}+\left|A_{\tau}\right|^{2}} \sin ^{2}\left[\sqrt{\varepsilon_{\tau}^{2}+\left|A_{\tau}\right|^{2}} \frac{d k}{d E} L \frac{a}{2}\right]
$$

where

$$
\frac{d k}{d E} \equiv \sqrt{\left|\frac{d k_{\tau}^{O}}{d E} \frac{d k_{\tau}^{I}}{d E}\right|}
$$

is proportional to the geometrical mean of the density of states. Since the dispersion lines are almost straight and parallel,

$$
\varepsilon_{\tau} \simeq\left(\frac{d k}{d E}\right)^{-1} \Delta \widetilde{k}_{\tau}
$$

Using Eqs. (28), (30), (36) and (37), we can transform Eq.(35) to

$$
\begin{aligned}
T_{\tau, \tau}^{\prime} & =\left(1-\left|\frac{\Delta \widetilde{k}_{\tau}}{\Delta k_{\tau}^{D, A}}\right|^{2}\right) \sin ^{2}\left(\frac{\Delta k_{\tau}^{D, A}}{2} L a\right) \\
& =\frac{x_{\tau}}{1+x_{\tau}} \sin ^{2}\left(\sqrt{1+x_{\tau}} \frac{\Delta \widetilde{k}_{\tau}}{2} L a\right)
\end{aligned}
$$

\section{B. Exact numerical calculations}

The scattering matrix method was used as an exact method..$^{21-23}$ We can also use the Green's function method, which is useful for including inelastic scattering, electron correlation and finite bias. ${ }^{24,25}$ Without these effects, however, the same transmission rate is obtained by both methods. ${ }^{26}$ There are advantages of using scattering matrix method compared to the Green's function method. One is the explicit relation to the wave function, and the other is that we can estimate the numerical error from the unitarity of the scattering matrix.

We can obtain only the propagating states from Eq. (13), but the evanescent wave states are also necessary for the exact calculation of the transmission rate. In order to obtain both the propagating and evanescent states, the transfer matrix $\Gamma_{\mu}$ is defined as

$$
\Gamma_{\mu} \equiv\left(\begin{array}{cccc}
0, & 1, & 0, & 0 \\
0, & 0, & 1, & 0 \\
0, & 0, & 0, & 1 \\
\tilde{Y}_{2}^{\mu}, & \tilde{Y}_{1}^{\mu}, & Y_{0}^{\mu}, & Y_{1}^{\mu}
\end{array}\right)
$$

where

$$
\begin{gathered}
Y_{j}^{\mu} \equiv-\left(P_{3,5}^{\mu, \mu}\right)^{-1} P_{3,3+j}^{\mu, \mu} \\
\tilde{Y}_{j}^{\mu} \equiv-\left(P_{3,5}^{\mu, \mu}\right)^{-1}{ }^{t} P_{3,3+j}^{\mu, \mu} .
\end{gathered}
$$

The matrix $P$ is defined as

$$
P_{j^{\prime}, j}^{\mu^{\prime}, \mu} \equiv H_{j^{\prime}, j}^{\mu^{\prime}, \mu,[\mathrm{fi}]}-\left(E+\Delta \varepsilon \delta_{\mu^{\prime}, \mu} \delta_{\mu, O}\right) B_{j^{\prime}, j}^{\mu^{\prime}, \mu,[\mathrm{fi}]} .
$$

Figure 6 shows the relation between structure [fi] and the matrixes $P$. The matrix $P^{D, D}$ is composed of $P^{I, I}, P^{O, I}, P^{I, O}$ and $P^{O, O}$ in the same way as Eq. (11).

The secular equation is represented by $\vec{e}_{j+1}=\Gamma_{\mu} \vec{e}_{j}$ where ${ }^{t} \vec{e}_{j} \equiv\left({ }^{t} \vec{d}_{j-2}^{\mu},{ }^{t} \vec{d}_{j-1}^{\mu},{ }^{t} \vec{d}_{j}^{\mu},{ }^{t} \vec{d}_{j+1}^{\mu}\right)$ with $\mu$ corresponding to $j$ as $(\mu=I, j \leq-2),(\mu=D, 3 \leq j \leq L-2)$ 
and $(\mu=O, L+3 \leq j)$. For the propagating waves, the eigenvector $\vec{u}$ and the eigenvalue $\lambda$ of the transfer matrix $\Gamma_{\mu}$ satisfying $\Gamma_{\mu} \vec{u}=\lambda \vec{u}$ and $\vec{e}_{j}=\lambda^{j} \vec{u}$ can be related to $k$ and $\vec{g}^{\mu}$ of Eq. (13) as $\lambda=e^{i k a}$ and ${ }^{t} \vec{u}=\left({ }^{t} \vec{g}^{\mu}, \lambda{ }^{t} \vec{g}^{\mu}, \lambda^{2}{ }^{t} \vec{g}^{\mu}, \lambda^{3}{ }^{t} \vec{g}^{\mu}\right)$. In the following, the dimension of $\vec{g}_{\tau}^{\mu}$ is denoted by $N_{\mu}\left(N_{I}=80, N_{O}=\right.$ $\left.160, N_{D}=240\right)$. The $4 N_{\mu}$ independent eigenvectors of $4 N_{\mu} \times 4 N_{\mu}$ matrix $\Gamma_{\mu}$ are labeled by $\tau$ as follows. For the propagating waves $\left(\left|\lambda_{\tau}^{\mu}\right|=1\right), \tau= \pm 1, \pm 2, \cdots, \pm N_{c}^{\mu}$. For the evanescent waves $\left(\left|\lambda_{\tau}^{\mu}\right| \neq 1\right), \tau= \pm\left(N_{c}^{\mu}+\right.$ $1), \pm\left(N_{c}^{\mu}+2\right), \cdots, \pm 2 N_{\mu}$. The sign of $\tau$ is chosen to be consistent with the propagation direction and decay direction when $|\tau| \leq N_{c}^{\mu}$ and $|\tau| \geq N_{c}^{\mu}+1$, respectively. Here, the channel number $N_{c}^{\mu}$ denotes half the number of independent propagating waves in region $\mu$. In the present paper, the energy $E$ is close to the neutral Fermi level, and thus $N_{c}^{I}=N_{c}^{O}=2$ and $N_{c}^{D}=4$.

The amplitude vector $\vec{d}_{j}$ is represented by

$$
\vec{d}_{j}^{\mu}=\sum_{\tau=-N_{\mu}}^{N_{\mu}}\left(\lambda_{\tau}^{\mu}\right)^{j} \vec{g}_{\tau}^{\mu} \gamma_{\tau}^{\mu}
$$

where $\tau \neq 0$ and the correspondence between $\mu$ and $j$ is represented by $(\mu=I, j \leq 0),(\mu=D, 1 \leq j \leq L)$ and $(\mu=O, L+1 \leq j)$.

In the periodic region $(\mu=I, j \leq-2),(\mu=D, 3 \leq$ $j \leq L-2)$ and $(\mu=O, L+3 \leq j)$, the secular equations can be represented by

$$
\vec{d}_{j+2}^{\mu}=\sum_{\Delta j=-2}^{-1} \tilde{Y}_{|\Delta j|}^{\mu} \vec{d}_{j+\Delta j}^{\mu}+\sum_{\Delta j=0}^{1} Y_{\Delta j}^{\mu} \vec{d}_{j+\Delta j}^{\mu}
$$

with Eqs. (40) and (41). In the transition region, the secular equations can be represented by

$$
\begin{gathered}
{ }^{t} Q_{1}^{I} \vec{c}_{-3}^{I}+Q_{0}^{I} \vec{c}_{-1}^{I}+q_{I D} \vec{c}_{1}^{D}=0 \\
{ }^{t} q_{I D} \vec{c}_{-1}^{I}+q_{0}^{\prime} \vec{c}_{1}^{D}+Q_{1}^{D} \vec{c}_{3}^{D}=0 \\
{ }^{t} Q_{1}^{D} \vec{c}_{L-3}^{D}+q_{0}^{\prime \prime} \vec{c}_{L-1}^{D}+q_{D O} \vec{c}_{L+1}^{O}=0 \\
{ }^{t} q_{D O} \vec{c}_{L-1}^{D}+Q_{0}^{O} \vec{c}_{L+1}^{O}+Q_{1}^{O} \vec{c}_{L+3}^{O}=0 .
\end{gathered}
$$

where the matrixes $Q$ and the vector $\vec{c}$ are defined as

$$
\begin{gathered}
\vec{c}_{j}^{\mu} \equiv\left(\begin{array}{c}
\vec{d}_{j}^{\mu} \\
\vec{d}_{j+1}^{\mu}
\end{array}\right), \\
Q_{0}^{\mu} \equiv\left(\begin{array}{cc}
P_{3,3}^{\mu, \mu}, & P_{3,4}^{\mu, \mu} \\
{ }^{t} P_{3,4}^{\mu, \mu}, & P_{3,3}^{\mu, \mu}
\end{array}\right), \\
Q_{1}^{\mu} \equiv\left(\begin{array}{cc}
P_{3, \mu}^{\mu, \mu}, & 0 \\
P_{3,4}^{\mu, \mu}, & P_{3,5}^{\mu, \mu}
\end{array}\right)
\end{gathered}
$$

and the matrixes $q$ are defined as

$$
\begin{aligned}
& q_{0}^{\prime} \equiv\left(\begin{array}{cc}
P_{1,1}^{D, D}, & P_{1,2}^{D, D} \\
{ }^{t} P_{1,2}^{D, D}, & P_{2,2}^{D, D}
\end{array}\right) \\
& q_{I D} \equiv\left(\begin{array}{cccc}
P_{3,5}^{I, I}, & P_{3,5}^{I, O}, & 0, & 0 \\
P_{3,4}^{I, I}, & P_{3,4}^{I, O}, & P_{3,5}^{I, I}, & P_{3,5}^{I, O}
\end{array}\right) \\
& q_{0}^{\prime \prime} \equiv\left(\begin{array}{cc}
P_{4,4}^{D, D}, & P_{4,5}^{D, D} \\
{ }^{t} P_{4,5}^{D, D}, & P_{5,5}^{D, D}
\end{array}\right) \\
& { }^{t} q_{D O} \equiv\left(\begin{array}{cccc}
{ }^{t} P_{3,5}^{I, O}, & { }^{t} P_{3,5}^{O, O}, & { }^{t} P_{3,4}^{I, O}, & { }^{t} P_{3,4}^{O, O} \\
0 & 0 & { }^{t} P_{3,5}^{I, O}, & { }^{t} P_{3,5}^{O, O}
\end{array}\right)
\end{aligned}
$$

Although the coefficients $\left\{\gamma_{\tau}^{I}\right\},\left\{\gamma_{\tau}^{O}\right\}$ and $\left\{\gamma_{\tau}^{D}\right\}$ in Eq. (43) can be chosen arbitrarily to satisfy Eq. (44), they are subject to the conditions expressed in Eqs. (45),(46),(47) and ,(48). Using Eqs. (43),(45),(46) and (49), we can obtain a $320 \times 320$ matrix $\sigma^{I D}$ satisfying $\left({ }^{t} \vec{\gamma}_{-}^{I},{ }^{t} \vec{\gamma}_{+}^{D}\right)=$ $\left({ }^{t} \vec{\gamma}_{+}^{I}{ }^{t} \vec{\gamma}_{-}^{D}\right)^{t} \sigma^{I D}$, where ${ }^{t} \vec{\gamma}_{ \pm}^{\mu}=\left(\gamma_{ \pm 1}^{\mu}, \gamma_{ \pm 2}^{\mu}, \cdots, \gamma_{ \pm N_{\mu}}^{\mu}\right)$. The matrix $\sigma^{D O}$ satisfying $\left({ }^{t} \vec{\gamma}_{-}^{D}, t \vec{\gamma}_{+}^{O}\right)=\left({ }^{t} \vec{\gamma}_{+}^{D},{ }^{t} \vec{\gamma}_{-}^{O}\right){ }^{t} \sigma^{D O}$ can be obtained in the same way. By eliminating $\vec{\gamma}_{ \pm}^{D}$ from these equations, we can obtain the matrix $\sigma^{I O}$ satisfying $\left({ }^{t} \vec{\gamma}_{-}^{I},{ }^{t} \vec{\gamma}_{+}^{O}\right)=\left({ }^{t} \vec{\gamma}_{+}^{I},{ }^{t} \vec{\gamma}_{-}^{O}\right)^{t} \sigma^{I O}$. The $4 \times 4$ scattering matrix $S^{I O}$ is obtained from the $240 \times 240$ matrix $\sigma^{I O}$ as $\left(S^{I O}\right)_{\tau, \tau^{\prime}}=\left(\sigma^{I O}\right)_{\tau, \tau^{\prime}}, S_{\tau+2, \tau^{\prime}}^{I O}=\sigma_{\tau+80, \tau^{\prime}}^{I O}, S_{\tau, \tau^{\prime}+2}^{I O}=$ $\sigma_{\tau, \tau^{\prime}+80}^{I O}$ and $S_{\tau+2, \tau^{\prime}+2}^{I O}=\sigma_{\tau+80, \tau^{\prime}+80}^{I O}$, where $\tau=1,2$ and $\tau^{\prime}=1,2$. From the scattering matrix, we can obtain $T_{\tau, \tau^{\prime}}=\left|S_{\tau+2, \tau^{\prime}}^{I O}\right|^{2}$.

The difference between ${ }^{t} S^{I O *} S^{I O}$ and the unit matrix is represented by

$$
\operatorname{err}\left(S^{I O}\right) \equiv \sum_{l_{1}=1}^{4} \sum_{l_{2}=1}^{4}\left|\left(\sum_{l_{3}=1}^{4} S_{l_{3}, l_{1}}^{I O *} S_{l_{3}, l_{2}}^{I O}\right)-\delta_{l_{1}, l_{2}}\right|^{2}
$$

When there is no numerical error, $S^{I O}$ must be unitary and Eq. (56) must be zero with the normalization shown in Appendix A. ${ }^{26,27}$ As the unitarity represents that the sum of transmission rate and reflection rate must be unity, the numerical error in the transmission rate is estimated to be Eq. (56).

\section{RESULTS}

Figure 7 shows the dispersion lines for (a) $\Delta \varepsilon=-0.015$ , (b) $\Delta \varepsilon=0$, and (c) $\Delta \varepsilon=0.020$ Ry. The relationship between $\Delta \varepsilon$ and $\varepsilon_{\tau}$ is shown by Eq. (16). At $k=0.65 \pi / a$, $\varepsilon_{-1}=-0.022+\Delta \varepsilon$ and $\varepsilon_{2}=-0.028+\Delta \varepsilon$. Crosses represent $E_{\tau}^{O}(k)$ and $E_{\tau}^{I}(k)$ without interlayer interaction. In Fig. 7 (c), $E_{-1}^{O}$ and $E_{-1}^{I}$ almost coincide with each other 
as $\varepsilon_{-1} \simeq 0$. The dispersion lines with interlayer interaction are shown by circles for the exact $E^{D}(k)$ and by solid lines for the approximate $E^{D, A}(k)$ defined by Eqs. (27), (28), (29) and (30). Since $\left|A_{2}\right| \ll\left|\varepsilon_{2}\right|, E_{2}^{O}$ and $E_{2}^{I}$ are almost the same as $E_{2}^{D, A}$ and $E_{4}^{D, A}$, respectively. In the symmetric bands specified by $\tau=-1$, on the other hand, the relatively large $\left|A_{-1}\right|$ causes a clear difference between the solid lines and the crosses. As $\left|A_{-1}\right|$ is almost constant, $E_{-1}^{I}$ and $E_{-1}^{O}$ are almost parallel to $E_{-1}^{D, A}$ and $E_{-3}^{D, A}$. The solid lines reproduce the band split of $\tau=-1$ channel, but underestimate that of $\tau=2$ channel.

Figures 8 and 9 shows $T_{1,1}$ and $T_{2,2}$, respectively, as a function of the number of unit cells $L$. The circles represent the exact transmission rate. The values of $\Delta \varepsilon$ in Figs. 8 and 9 are the same as in Fig. 7. Because $L$ must be larger than three in the exact calculation with Eqs. (46) and (47), $L \geq 4$ in Figs. 8 and 9. Triangles in Fig. 9 represent Eq. (56) showing the numerical errors of $T_{1,1}+T_{2,2}+T_{2,1}+T_{1,2}$ in the exact calculation. The average and maximum of Eq. (56) are $4.9 \times 10^{-4}$ and $1.4 \times 10^{-3}$. The magnitude of $T_{1,2}+T_{2,1}$ obtained from the numerical calculation is also $10^{-4} \sim 10^{-3}$. This indicates that $T_{1,2}+T_{2,1}$ cannot be discussed with sufficient accuracy. Thus we focus our discussion on $T_{1,1}$ and $T_{2,2}$. Although the interlayer Hamiltonian could cause a pseudo-gap in the energy band of the DWNT, ${ }^{28}$ there is no pseudo-gap at the energy $E=-0.4$ Ry chosen here. The circles shows a rapid oscillation superposed on a slow oscillation. The rapid oscillation is due to a standing wave caused by a large intralayer reflection at the open edges $j=1, L$ in structure [td]. As the wavenumber $k$ is close to $\pm 2 \pi /(3 a)$, the period of the rapid oscillation is close to three. In order to see the slow oscillation of the exact $T(L)$,

$$
T^{\text {ave }}(L) \equiv \frac{T(L-1)+T(L)+T(L+1)}{3}
$$

is shown by the dot-dashed lines. In order to estimate the edge effect, $q_{0}^{\prime}$ in Eq. (46) and $q_{0}^{\prime \prime}$ in Eq. (47) are replaced by $Q_{0}^{D}$. The exact $T_{1,1}$ and $T_{1,1}^{a v e}$ with this replacement are shown by crosses and dashed lines, respectively, in Fig. 8. Agreement between the dashed line and dotdashed line indicates that the slow oscillation defined by (57) is not sensitive to the detail of the edge modeling. Though crosses and dashed lines are omitted in Fig. 9, it is also confirmed that the two edge models show similar $T_{2,2}^{\text {ave }}$.

The thick solid lines represent $T^{\prime}$ defined by Eq. (38), while the thin solid lines represent Eq.(38) where $E \widetilde{B}^{O, I}$ in Eq.(23) is replaced by zero, i.e., the interlayer overlap matrix is set to zero. The thin solid lines are shown only for the first period. Though the thin solid lines are close to $T^{\text {ave }}$ for the small $L$, the thick solid lines qualitatively reproduce $T^{\text {ave }}$ for the wider range of $L$. Thus we concentrate our discussion to the thick solid lines below. The effective interlayer interaction of the symmetric state $A_{1}$ is much larger than that of the anti-symmetric state $A_{2}$. This is the reason why $T_{1,1} \gg T_{2,2}$. In the derivation of Eq. (35) shown in Ref. ${ }^{15}$, the interlayer Hamiltonian is the same as that for structure [td], while the intralayer Hamiltonian is the same as that for structure [in]. Thus, Eq. (35) does not include the strong intralayer reflection at the edge $j=1, L$ of structure [td]. This is why the rapid oscillation does not appear in Eq. (38).

The period of the exact $T^{\text {ave }}$ is close to $2 \pi / \Delta k^{D}$ where $\Delta k_{\tau}^{D}$ denotes the exact band split of channel $\tau$ with interlayer interaction. Since $\Delta k_{1}^{D, A} \simeq \Delta k_{1}^{D}$, the thick solid line and the dot-dashed line have almost the same period in Fig. 8. On the other hand, the thick solid lines show the longer period than the dot-dashed lines in Fig. 9 because $\Delta k_{2}^{D, A}<\Delta k_{2}^{D}$. Nevertheless the peak height of $T^{\text {ave }}$ is qualitatively reproduced by $x /(1+x)$ of Eq. (38) both in Fig. 8 and in Fig. 9.

\section{DISCUSSION}

In the present paper, we consider the differential conductance $d I / d V$ at zero bias voltage $V=0$. We have to use more sophisticated numerical methods to obtain the effect of the finite $V$ on the current $I{ }^{25}$ If the rigid band picture is effective, however, the current $I$ for finite $V$ can be approximated by

$$
I=\frac{e}{h} \int_{E_{F}}^{E_{F}+e V} T(E, \varepsilon+e V) d E
$$

where $E_{F}$ is the Fermi level of tube $O$ and the interlayer transmission rate is represented by $T(E, \varepsilon)$ as a function of the energy $E$ and the intrinsic shift $\varepsilon$.

The effective interlayer interaction $A$ defined by Eqs. (23) enables us to analyze the effects of the interlayer Hamiltonian on the transmission rate. For example, we can discuss the difference between the TB used in Ref. ${ }^{15}$ and the SIESTA used here. The longer cut-off distance of the interlayer Hamiltonian decreases $\left|A_{2}\right|$ as was discussed in Ref. ${ }^{15}$. The cut-off distance for SIESTA is much larger than that for $\mathrm{TB}$ as seen in Fig. 5. This is why $\left|A_{2} / A_{1}\right|$ and $T_{2,2}$ for SIESTA are negligible compared to those for TB. Using the perturbation formulas, we can distinguish the effects of the intralayer Hamiltonian $H^{O, O}, H^{I, I}$ from those of the interlayer Hamiltonian $H^{O, I}$. Rigorously speaking, the interlayer interaction influences $H^{I, I}$ and $H^{O, O}$ in the self consistent calculation of structure [fi] for the building block procedure. Nevertheless, $H^{I, I}$ and $H^{O, O}$ are approximately considered as the Hamiltonian of isolated SWNTs. As long as a real space basis such as an AO basis is used, the calculation methods in the present paper can be applied to various other first-principles calculation codes.

Equation (38) is effective both in the SIESTA Hamiltonian and in the TB Hamiltonian. We have to be careful that the effective interlayer interaction (23) must include the interlayer overlap matrix $B^{O, I}$ that is absent in 
Ref. $^{15}$. Using Eq.(38), we can predict the amplitude of $T^{\text {ave }}(L)$ as seen in Figs. 8 and 9. Though the precision of Eq.(38) is lower in Fig. 9, the qualitative dependence on $\varepsilon$ is reproduced. The period of $T_{1,1}^{a v e}(L)$ is also reproduced well, but that of $T_{2,2}^{a v e}$ is overestimated. It is common to TB and SIESTA that Eq.(38) is more effective for $T_{1,1}$ than for $T_{2,2}$. The period $2 \pi / \Delta k$ has been discussed in other papers, but the amplitude formula $x_{\tau} /\left(1+x_{\tau}\right)$ in Eq.(38) is proposed firstly here. Note that the dimensionless parameter $x_{\tau}$ is defined by Eq. (30) without a fitting parameter. Owing to the linear dispersion, the amplitude of $T_{1,1}^{\prime}$ are represented by the energy ratios as

$$
1-\left|\frac{\Delta \tilde{k}_{1}}{\Delta k_{1}^{D}}\right|^{2}=1-\left|\frac{\varepsilon_{1}}{E_{3}^{D}-E_{1}^{D}}\right|^{2}
$$

When $\left|\varepsilon_{1}\right| /\left(E_{3}^{D}-E_{1}^{D}\right) \simeq 1$, Eq.(59) is close to $2\left(E_{3}^{D}-E_{1}^{D}-\left|\varepsilon_{1}\right|\right) /\left|\varepsilon_{1}\right|$. Since $E_{3}^{D}-E_{1}^{D}-\left|\varepsilon_{1}\right|$ is the energy shift caused by the interlayer interaction, its ratio to $\left|\varepsilon_{1}\right|$ can be considered as the Thouless number. ${ }^{29}$ Author expects that Eq. (38) represent the generalized Thouless number analysis and can be applied to other related systems with commensurate interlayer configurations.

\section{APPENDIX A: RELATION BETWEEN THE NORMALIZATION AND PROBABILITY FLOW}

The probability flow $J\left(\gamma^{\prime} \mid \gamma\right)$ from orbital $\gamma=$ $(\mu, l, m, \alpha, j)$ to orbital $\gamma^{\prime}=\left(\mu^{\prime}, l^{\prime}, m^{\prime}, \alpha^{\prime}, j^{\prime}\right)$ is represented by

$$
J\left(\gamma^{\prime} \mid \gamma\right) \equiv \operatorname{Im}\left[\left(H_{\gamma^{\prime}, \gamma}-E B_{\gamma^{\prime}, \gamma}\right) d_{\gamma^{\prime}}^{*} d_{\gamma}\right]
$$

where $H, B$ and $E$ represent the Hamiltonian matrix, the overlap matrix and the energy, respectively. Equation (A1) satisfies the two necessary conditions for stationary flow: 'direction of flow' represented by $J\left(\gamma \mid \gamma^{\prime}\right)=$ $-J\left(\gamma^{\prime} \mid \gamma\right)$ and 'conservation of probability ' represented by

$$
\sum_{\gamma} J\left(\gamma^{\prime} \mid \gamma\right)=0
$$

Equation (A2) is derived from the secular Equation $\sum_{\gamma}\left(H_{\gamma^{\prime}, \gamma}-E B_{\gamma^{\prime}, \gamma}\right) d_{\gamma}=0$. Using Eq. (A1), the probability flow $I_{\text {tot }}(j)$ through the cross section at $z=\left(j-\frac{1}{2}\right) a$ is represented by

$$
I_{t o t}(j)=I_{j, j-1}^{\mu}+I_{j+1, j-1}^{\mu}+I_{j, j-2}^{\mu}
$$

where

$$
I_{j, j^{\prime}}^{\mu} \equiv \sum_{l, m, \alpha} \sum_{l^{\prime}, m^{\prime}, \alpha^{\prime}} J\left(\mu, l, m, \alpha, j \mid \mu, l^{\prime}, m^{\prime}, \alpha^{\prime}, j^{\prime}\right) .
$$

Corresponding to $M=2$ in Eq. (12), $I_{j, j^{\prime}}^{\mu}=0$ when $\left|j-j^{\prime}\right| \geq 3$. Using Eqs. (42), (43), (51), (A1) and (A4), Eq. (A3) can be expanded as

$$
I_{\text {tot }}(j)=\sum_{\tau=-N_{\mu}}^{N_{\mu}} \sum_{\tau^{\prime}=\tau}^{N_{\mu}}\left(1-\frac{\delta_{\tau, \tau^{\prime}}}{2}\right)\left[\eta_{\tau^{\prime}, \tau}^{\mu}+\eta_{\tau, \tau^{\prime}}^{\mu}\right]
$$

where $\tau \neq 0, \tau^{\prime} \neq 0$,

$$
\begin{gathered}
\eta_{\tau^{\prime}, \tau}^{\mu} \equiv \operatorname{Im}\left[\left(\lambda_{\tau^{\prime}}^{\mu *} \lambda_{\tau}^{\mu}\right)^{j} Z_{\tau^{\prime}, \tau}^{\mu} \gamma_{\tau^{\prime}}^{\mu *} \gamma_{\tau}^{\mu}\right] \\
Z_{\tau^{\prime}, \tau}^{\mu} \equiv\left(\lambda_{\tau}^{\mu}\right)^{-2}\left({ }^{t} \vec{f}_{\tau^{\prime}}^{\mu *}\right){ }^{t} Q_{1}^{\mu} \vec{f}_{\tau}^{\mu}
\end{gathered}
$$

and

$$
\vec{f}_{\tau}^{\mu} \equiv\left(\begin{array}{c}
\vec{g}_{\tau}^{\mu} \\
\lambda_{\tau}^{\mu} \vec{g}_{\tau}^{\mu}
\end{array}\right)
$$

In Eq. (A5), the correspondence between $\mu$ and $j$ is represented by $(\mu=I, j \leq-2),(\mu=D, 3 \leq j \leq L-2)$ and $(\mu=O, L+3 \leq j)$.

On the other hand, we can obtain

$$
\left(\left(\lambda_{\tau}^{\mu}\right)^{-2 t} Q_{1}^{\mu}+Q_{0}^{\mu}+\left(\lambda_{\tau}^{\mu}\right)^{2} Q_{1}^{\mu}\right) \vec{f}_{\tau}^{\mu}=0
$$

from the secular equations. Multiplying Eq. (A9) by ${ }^{t} \vec{f}_{\tau^{\prime}}^{\mu *}$, we can obtain

$$
\left(\lambda_{\tau}^{\mu} \lambda_{\tau^{\prime}}^{\mu *}\right)^{2} Z_{\tau, \tau^{\prime}}^{\mu *}+Z_{\tau^{\prime}, \tau}^{\mu}+{ }^{t} \vec{f}_{\tau^{\prime}}^{\mu *} Q_{0}^{\mu} \vec{f}_{\tau}^{\mu}=0
$$

Exchanging $\tau$ and $\tau^{\prime}$ in the complex conjugate of Eq. (A10), we can also obtain

$$
\left(\lambda_{\tau}^{\mu} \lambda_{\tau^{\prime}}^{\mu *}\right)^{2} Z_{\tau^{\prime}, \tau}^{\mu}+Z_{\tau, \tau^{\prime}}^{\mu *}+{ }^{t} \vec{f}_{\tau^{\prime}}^{\mu *} Q_{0}^{\mu} \vec{f}_{\tau}^{\mu}=0
$$

When the condition

$$
\left(\lambda_{\tau}^{\mu} \lambda_{\tau^{\prime}}^{\mu *}\right)^{4}=1
$$

does not hold, we can show

$$
Z_{\tau^{\prime}, \tau}^{\mu}=Z_{\tau, \tau^{\prime}}^{\mu *}=\frac{-{ }^{t} \vec{f}_{\tau^{\prime}}^{\mu *} Q_{0}^{\mu} \vec{f}_{\tau}^{\mu}}{\left(\lambda_{\tau}^{\mu} \lambda_{\tau^{\prime}}^{\mu *}\right)^{2}+1}
$$

from Eqs. (A10) and (A11) . Thus, $\eta_{\tau^{\prime}, \tau}^{\mu}+\eta_{\tau, \tau^{\prime}}^{\mu}$ has a nonzero value only when the condition (A12) holds. Under the condition (A12), we only have to consider three cases (i) $\left|\lambda_{\tau}^{\mu}\right|=\left|\lambda_{\tau^{\prime}}^{\mu}\right|^{-1} \neq 1$, (ii) $\tau=\tau^{\prime},\left|\lambda_{\tau}^{\mu}\right|=1$, and (iii) $\tau \neq \tau^{\prime},\left|\lambda_{\tau}^{\mu}\right|=\left|\lambda_{\tau^{\prime}}^{\mu}\right|=1$. When $\left|\lambda_{\tau_{1}}^{O}\right|>1$ and $\left|\lambda_{\tau_{2}}^{I}\right|<1$, $\gamma_{\tau_{1}}^{O}=\gamma_{\tau_{2}}^{I}=0$ because finite values of $\gamma_{\tau_{1}}^{O}$ and $\gamma_{\tau_{2}}^{I}$ cause the divergence of $\left|\vec{d}_{j}^{\mu}\right|$ at $j=\infty$ and $j=-\infty$, respectively. Thus, $\gamma_{\tau^{\prime}}^{\mu *} \gamma_{\tau}^{\mu}$ in Eq. (A6) must be zero in case (i). In case (iii), $\left(\lambda_{\tau}^{\mu}\right)^{4}$ and $\left(\lambda_{\tau^{\prime}}^{\mu}\right)^{4}$ are accidentally degenerate. Since this degeneracy is lifted by an infinitesimal change in the energy $E$, case (iii) can be excluded. Since $\eta_{\tau^{\prime}, \tau}^{\mu}+\eta_{\tau, \tau^{\prime}}^{\mu}$ is nonzero only in case (ii), Eq. (A5) is represented by

$I_{\text {tot }}(j)=\sum_{\tau=1}^{N_{c}^{\mu}} \operatorname{Im}\left(Z_{\tau, \tau}^{\mu}\right)\left|\gamma_{\tau}^{\mu}\right|^{2}+\operatorname{Im}\left(Z_{-\tau,-\tau}^{\mu}\right)\left|\gamma_{-\tau}^{\mu}\right|^{2}$. 
Equations (A5) and (A6) do not depend on $j$ because $\lambda_{\tau^{\prime}}^{\mu *} \lambda_{\tau}^{\mu}=\lambda_{\tau}^{\mu *} \lambda_{\tau}^{\mu}=\left|\lambda_{\tau}^{\mu}\right|^{2}=1$ in case (ii).

When the vectors $\vec{f}_{\tau}^{\mu}$ are normalized as $\operatorname{Im}\left(Z_{|\tau|,|\tau|}\right)=1$ and $\operatorname{Im}\left(Z_{-|\tau|,-|\tau|}\right)=-1$, we can show

$$
\begin{aligned}
I_{\text {tot }} & =\sum_{\tau=1}^{N_{c}^{I}}\left(\left|\gamma_{\tau}^{I}\right|^{2}-\left|\gamma_{-\tau}^{I}\right|^{2}\right)=\sum_{\tau=1}^{N_{c}^{D}}\left(\left|\gamma_{\tau}^{D}\right|^{2}-\left|\gamma_{-\tau}^{D}\right|^{2}\right) \\
& =\sum_{\tau=1}^{N_{c}^{O}}\left(\left|\gamma_{\tau}^{O}\right|^{2}-\left|\gamma_{-\tau}^{O}\right|^{2}\right) .
\end{aligned}
$$

Here, conservation of probability guarantees that $I_{t o t}$ is common to regions $I, D$, and $O$. Eq. (A15) is equivalent to ${ }^{t} S^{I D *} S^{I D}=1,{ }^{t} S^{D O *} S^{D O}=1$ and ${ }^{t} S^{I O *} S^{I O}=1$.

\section{APPENDIX B: RELATION BETWEEN THE NORMALIZATION AND GROUP VELOCITY}

From Eqs. (13) and (21), we can obtain

$$
b_{\tau}^{\mu} E_{\tau}^{\mu}={ }^{t} \vec{g}_{\tau}^{\mu *} \widetilde{H}^{\mu, \mu} \vec{g}_{\tau}^{\mu} .
$$

Differentiating Eq. (B1) with respect to $k$,

$$
\begin{aligned}
b_{\tau}^{\mu} \frac{d E_{\tau}^{\mu}}{d k}= & -\frac{d b_{\tau}^{\mu}}{d k} E_{\tau}^{\mu}+\frac{d^{t} \vec{g}_{\tau}^{\mu *}}{d k} \widetilde{H}^{\mu, \mu} \vec{g}_{\tau}^{\mu} \\
& +{ }^{t} \vec{g}_{\tau}^{\mu *} \frac{d \widetilde{H}^{\mu, \mu}}{d k} \vec{g}_{\tau}^{\mu}+{ }^{t} \vec{g}_{\tau}^{\mu *} \widetilde{H}^{\mu, \mu} \frac{d \vec{g}_{\tau}^{\mu}}{d k} .
\end{aligned}
$$

Using Eqs. (12),(13),(21) and (42), Eq. (B2) is represented by

$$
\begin{aligned}
b_{\tau}^{\mu} \frac{d E_{\tau}^{\mu}}{d k} & ={ }^{t} \vec{g}_{\tau}^{\mu *}\left[\frac{d \widetilde{H}^{\mu, \mu}}{d k}-E_{\tau}^{\mu} \frac{d \widetilde{B}^{\mu, \mu}}{d k}\right] \vec{g}_{\tau}^{\mu} \\
& =2 \operatorname{Re}\left[{ }^{t} \vec{g}_{\tau}^{\mu *} \sum_{\Delta j=1}^{2} P_{3,3+\Delta j}^{\mu, \mu}\left(\frac{d}{d k} e^{i k a j}\right) \vec{g}_{\tau}^{\mu}\right] .
\end{aligned}
$$

From Eqs. (51),(A7) and (A8), Eq. (B3) is represented by

$$
b_{\tau}^{\mu} \frac{d E_{\tau}^{\mu}}{d k}=2 a \operatorname{Im}\left(Z_{\tau, \tau}^{\mu}\right)
$$

With Eqs. (23), (36) and (B4), Eq. (30) can be represented by

$$
x_{\tau}=\frac{\left|{ }_{t_{\tau}}^{O}\left(k_{\tau}^{O}\right)^{*} \widetilde{P}^{O, I}\left(k_{\tau}^{I}\right) \vec{g}_{\tau}^{I}\left(k_{\tau}^{I}\right)\right|^{2}}{\left|\operatorname{Im}\left(Z_{\tau, \tau}^{O}\right) \operatorname{Im}\left(Z_{\tau, \tau}^{I}\right)\right| a^{2} \Delta \widetilde{k}_{\tau}^{2}} .
$$

When the normalization $\left|\operatorname{Im}\left(Z_{\tau, \tau}^{O}\right)\right|=\left|\operatorname{Im}\left(Z_{\tau, \tau}^{I}\right)\right|=1$ is used,

$$
x_{\tau}=\frac{\left|{ }_{g_{\tau}}^{O}\left(k_{\tau}^{O}\right)^{*} \widetilde{P}^{O, I}\left(k_{\tau}^{I}\right) \vec{g}_{\tau}^{I}\left(k_{\tau}^{I}\right)\right|^{2}}{a^{2}\left(k_{\tau}^{O}-k_{\tau}^{I}\right)^{2}} .
$$

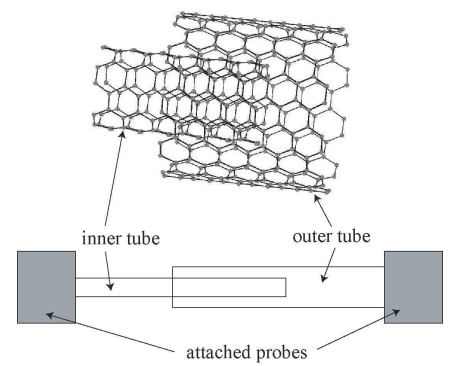

FIG. 1. TDWNT

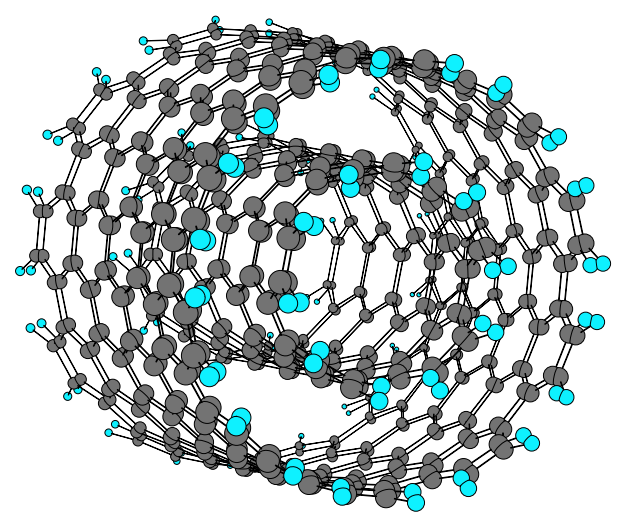

FIG. 2. Structure [fi] superimposed on its initial structure.
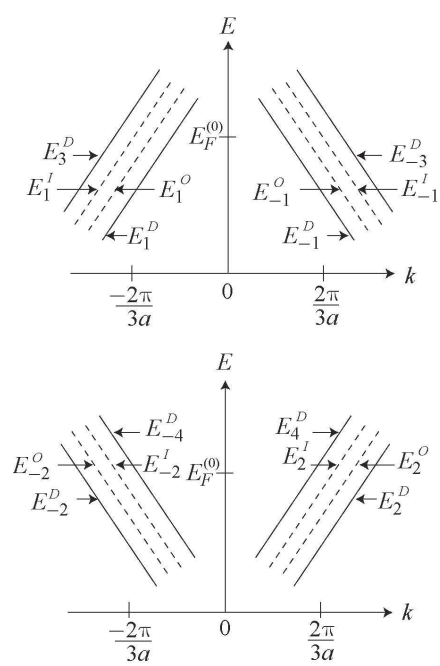

FIG. 3. The dispersion lines near the neutral Fermi level $E_{F}^{(0)}$ are numbered based on the index $\tau$ under the conditions $E_{|\tau|}^{\mu}\left((-1)^{\tau} \frac{2 \pi}{3 a}\right)=E_{-|\tau|}^{\mu}\left(-(-1)^{\tau} \frac{2 \pi}{3 a}\right) \simeq E_{F}^{(0)}$ and $\tau \frac{d E_{\tau}^{\mu}}{d k}>0$. 


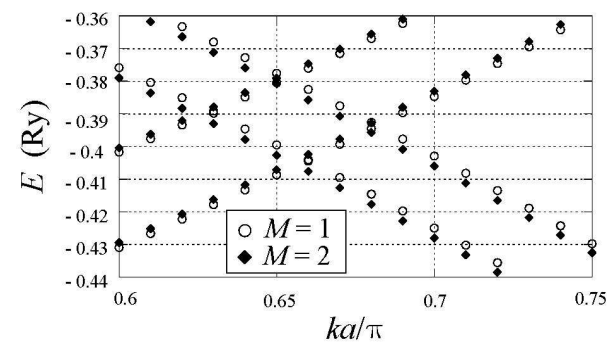

FIG. 4. The dispersion lines without interlayer interaction for $M=1$ (open circles) and $M=2$ (closed diamonds), where $M$ denotes the integer parameter of Eq. (12).

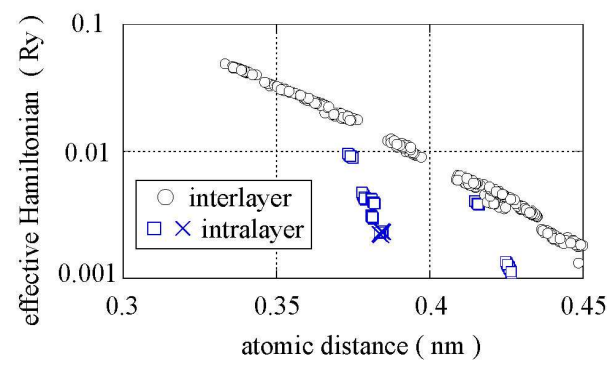

FIG. 5. Effective Hamiltonian elements defined by Eq. (14).
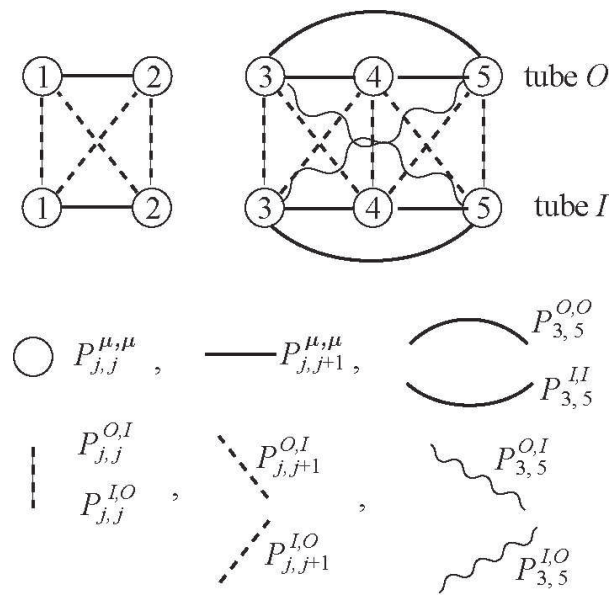

FIG. 6. Schema for the matrix $P$.
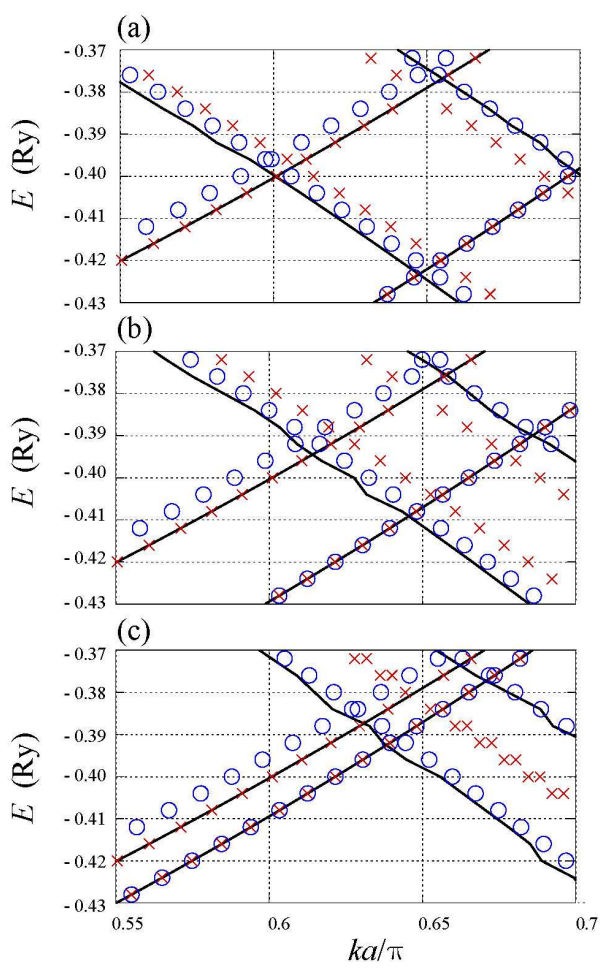

FIG. 7. The dispersion lines for (a) $\Delta \varepsilon=-0.015$ (b) $\Delta \varepsilon=$ 0 and (c) $\Delta \varepsilon=0.020$ Ry. At $k=0.65 \pi / a, \varepsilon_{-1}=-0.022+\Delta \varepsilon$ and $\varepsilon_{2}=-0.028+\Delta \varepsilon$. The crosses represent $k_{\tau}^{O}(E)$ and $k_{\tau}^{I}(E)$ without interlayer interaction. The dispersion lines with interlayer interaction are shown by circles for the exact $k^{D}(E)$ and by solid lines for the approximate $k^{D, A}(E)$. The wavenumber $k$ is calculated for the discrete energies $E=-0.428+0.004 j$ where $j=0,1,2, \cdots, 14$. 
(a)

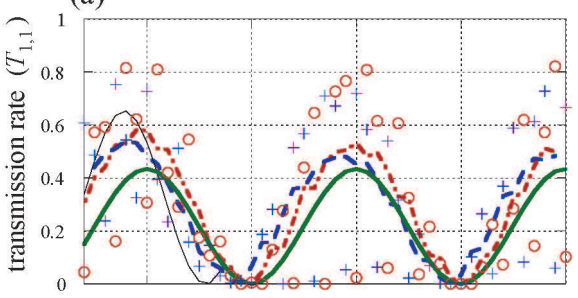

(b)

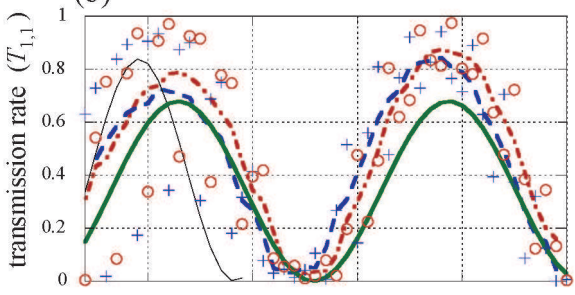

(c)

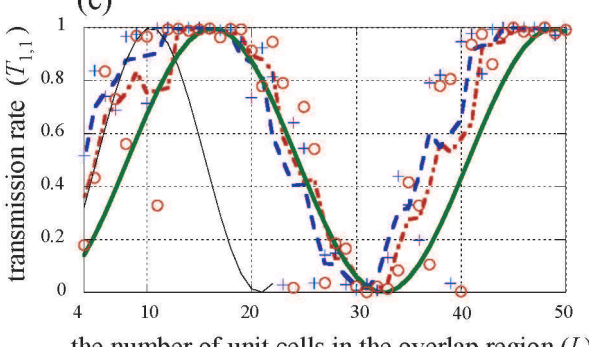

the number of unit cells in the overlap region $(L)$

FIG. 8. The transmission rate $T_{1,1}$ at an energy $E=-0.4$ Ry. The values of $\Delta \varepsilon$ are the same as in Fig. 7. The circles represent the exact $T_{1,1}$ with Eqs. (46) and (47). The crosses represent the exact $T_{1,1}$ when $q_{0}^{\prime}$ and $q_{0}^{\prime \prime}$ in Eqs. (46) and (47) are replaced by $Q_{0}^{D}$. The average defined by $T^{\text {ave }}(L)=(T(L-1)+T(L)+T(L+1)) / 3$ are shown by the dot-dashed and dashed lines for the circles and crosses, respectively. The approximate formula (38) is shown by thick solid lines. The thin solid represent Eq.(38) where $E \widetilde{B}^{O, I}$ in Eq.(23) is replaced by zero, i.e., the interlayer overlap matrix is set to zero. The thin solid lines are shown only for the first period. (a)

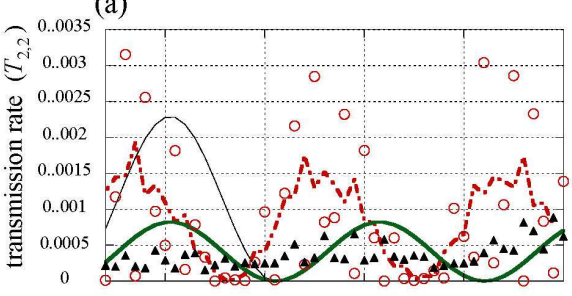

(b)

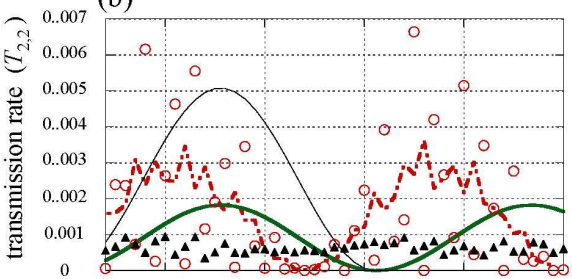

(c)

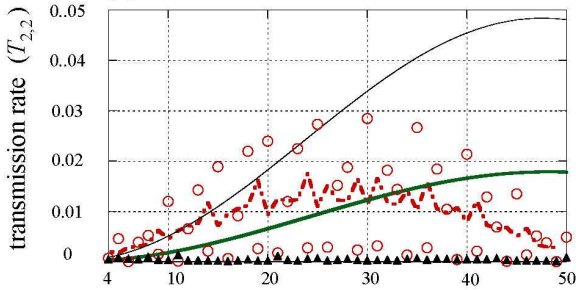

the number of unit cells in the overlap region $(L)$

FIG. 9. The same calculation as in Fig. 8 for the transmission rate $T_{2,2}$. The crosses and dashed lines are omitted. The triangles show Eq. (56).

${ }^{1}$ R. Saito, G. Dresselhaus, and M. S. Dresselhaus, Physical Propertiesof Carbon Nanotubes (Imperial College Press, London,1998).

2 J.-C. Charlier, X. Blase, and S. Roche, Rev. Mod. Phys. 79, 677 (2007).

${ }^{3}$ N. Hamada, S. I. Sawada, and A. Oshiyama, Phys. Rev. Lett. 68, 1579 (1992); J. W. Mintmire, B. I. Dunlap, and C. T. White, ibid. 68, 631 (1992); R. Saito, M. Fujita, G. Dresselhaus, and M. S. Dresselhaus, Phys. Rev. B 46, 1804 (1992).

${ }^{4}$ S. J. Tans, A. R. M. Verschueren and C. Dekker, Nature 393, 49 (1998); A. Bachtold, P. Hadley, T. Nakanishi and C. Dekker, Science 294, 1317 (2001); A. Rochefort, M. D. Ventra and P. Avouris, Appl. Phys. Lett. 78, 2521 (2001); R. Martel, V. Derycke, C. Lavoie, J. Appenzeller, K. K. Chan, J. Tersoff, and Ph. Avouris, Phys. Rev. Lett. 87, 256805 (2001).

${ }^{5}$ Z. Yao, Henk W. Ch. Postma, L. Balents and C. Dekker, Nature, 402, 273 (1999); P. G. Collins, A. Zettl, H. Bando, A. Thess, R. E. Smalley, Science 278 , 100, (1997); A. A. Odintsov, Phys. Rev. Lett. 85, 150 (2000); R. Tamura, Phys. Rev. B 64, R201404 (2001).

${ }^{6}$ M. S. Fuhrer, J. Nyg. rd, L. Shih, M. Forero, Y.-G. Yoon, M. S. C. Mazzoni, H. J. Choi, J. Ihm, S. G. Louie, A. Zettl 
and P. L. McEuen Science 288, 494 (2000).

${ }^{7}$ R. Saito, G. Dresselhaus, and M. S. Dresselhaus, Phys. Rev. B 53, 2044 (1996); V. Meunier, L. Henrard, and Ph. Lambin, ibid. 57, 2586 (1998); L. Chico, L. X. Benedict, S. G. Louie, and M. L. Cohen, ibid. 54, 2600 (1996); B. I. Dunlap, ibid. 46, 1933 (1992); J. C. Charlier, T. W. Ebbesen, and Ph. Lambin, ibid. 53, 11108 (1996); L. Chico, Vincent H. Crespi, Lorin X. Benedict, Steven G. Louie, and Marvin L. Cohen, Phys. Rev. Lett. 76, 971 (1996); R. Tamura, Phys. Rev. B 67, R121408 (2003); H. Matsumura and T. Ando, J. Phys. Soc. Jpn. 67, 3542 (1998); A. V. Krasheninnikov, K. Nordlund, J. Keinonen, F. Banhart, Phys. Rev. B 66, 245403 (2002); F. Cleri, P. Keblinski, I. Jang and S. B. Sinnott, Phys. Rev. B 69, R121412 (2004); F. Y. Meng, S. Q. Shi, D. S. Xu and R. Yang, Phys. Rev. B 70, 125418 (2004); H. T. Yang, J. W. Chen, L. F. Yang, and J. Dong, Phys. Rev. B 71, 073401 (2005); S. Dag, R. T. Senger and S. Ciraci, Phys. Rev. B 70, 205407 (2004); M. Terrones, F. Banhart, N. Grobert, J.-C. Charlier, H. Terrones and P. M. Ajayan, Phys. Rev. Lett. 89, 075505 (2002).

${ }^{8}$ T. Nakanishi and T. Ando, J. Phys. Soc. Jpn. 70, 1647 (2001); A. A. Maarouf and E. J. Mele, Phys. Rev. B 83, 045402 (2011); Y.-G. Yoon, M. S. C. Mazzoni, H. J. Choi, J. Ihm and S. G. Louie, Phys. Rev. Lett. 86, 688 (2001); B. G. Cook, P. Dignard and K. Varga, Phys. Rev. B 83, 205105 (2011).

${ }^{9}$ J. Cumings and A. Zettl, Science 289, 602 (2000); A. Kis, K. Jensen,S. Aloni, W. Mickelson, and A. Zettl, Phys. Rev. Lett. 97, 025501 (2006); S. Akita and Y. Nakayama, J. J. Appl. Phys. 42, 4830 (2003); M. Nakajima, S. Arai, Y. Saito, F. Arai and T. Fukuda, ibid. 46, L1035 (2007); W. Zhang, Z. Xi, G. Zhang, C. Li, and D. Guo, Phys. Chem. Lett. 112, 14714 (2008).

${ }^{10}$ J. Cumings and A. Zettl, Phys. Rev. Lett. 93, 086801 (2004); S. Akita and Y. Nakayama, J. J. Appl. Phys. 43, 3796 (2004).

${ }^{11}$ B. Bourlon, C. Miko, L. Forro, D. C. Glattli and A. Bachtold, Phys. Rev. Lett. 93, 176806 (2004).

12 J. Servantie and P. Gaspard, Phys. Rev. B 73, 125428 (2006); Phys. Rev. Lett. 91, 185503 (2003); Q. Zheng and Q. Jiang, Phys. Rev. Lett. 88, 045503 (2002); S. B. Legoas, V. R. Coluci, S. F. Braga, P. Z. Coura, S. O. Dantas, and D. S. Galvao, ibid. 90, 055504 (2003); W. Guo, Y. Guo, H. Gao, Q. Zheng and W. Zhong. ibid. 91, 125501 (2003); P. Tangney, M. L. Cohen, and S. G. Louie, ibid. 97, 195901 (2006); Q. Zheng, J. Z. Liu and Q. Jiang, Phys. Rev. B 65, 245409 (2002); J. W. Kang and O. K. Kwon Appl. Sur. Sci. 258, 2014 (2012).

${ }^{13}$ Q. Yan, G. Zhou, S. Hao, J. Wu, and W. Duan, Appl. Phys. Lett. 88, 173107 (2006); S. Uryu and T. Ando, Phys. Rev. B 76, 155434 (2007);72, 245403 (2005); A. Hansson and S. Stafstrom, Phys. Rev. B, 67, 075406 (2003); I. M. Grace, S. W. Bailey, and C. J. Lambert, Phys. Rev. B, 70, 153405 (2004); M. A. Tunney and N. R. Cooper, Phys. Rev. B 74, 075406 (2006).

${ }^{14}$ D.-H. Kim and K. J. Chang, Phys. Rev. B, 66, 155402 (2002); Y.-J. Kang, K. J. Chang, and Y.-H. Kim, Phys. Rev. B 76, 205441 (2007); C. Buia, A. Buldum, and J. P. Lu, Phys. Rev. B, 67, 113409 (2003); R. Tamura, Y. Sawai, and J. Haruyama, Phys. Rev. B 72, 045413 (2005).

${ }^{15}$ R. Tamura, Phys. Rev. B 82, 035415 (2010).

${ }^{16}$ K. S. Novoselov, A. K. Geim, S. V. Morozov, D. Jiang, Y. Zhang, S. V. Dubonos, I. V. Grigorieva, A. A. Firsov, Science 306, 666 (2004).

17 J. Nilsson, A. H. C. Neto, F. Guinea and N. M. R. Peres, Phys. Rev. B 76, 165416 (2007); J. W. Gonzalez, H. Santos, M. Pacheco, L. Chico and L. Brey, Phys. Rev. B 81, 195406 (2010); T. Nakanishi, M. Koshino and T. Ando, Phys. Rev. B 82, 125428 (2010).

18 J. M. Soler, E. Artacho, J. D. Gale, A. Garcia, J. Junquera, P. Ordejon, and D. Sanchez-Portal, J. Phys.: Condens. Matter 14, 2745 (2002); P. Ordejon, E. Artacho, and J. M. Soler, Phys. Rev. B 53, R10441 (1996).

${ }^{19}$ Emilio Artacho, Jose M. Cela,Julian Gale, Alberto Garcia,Javier Junquera, Richard M. Martin, Pablo Ordejon, Daniel Sanchez-Portal and Jose M. Soler, SIESTA (http://www.icmab.es/dmmis/leem/siesta).

${ }^{20}$ In the input file for the generation of the pseudopotential, following items are used. The type of exchange-correlation is Ceperley-Alder. The generation scheme is improved Troullier-Martins. The core radii for the carbon $2 \mathrm{~s}, 2 \mathrm{p}, 3 \mathrm{~d}$, 4f orbitals are 1.50, 1.54, 1.50 and $1.50 \mathrm{bohr}$, respectively. Those for the hydrogen $1 \mathrm{~s}, 2 \mathrm{p}, 3 \mathrm{~d}$, 4 f orbitals are $1.25 \mathrm{bohr}$.

${ }^{21}$ J. Feilhauer and M. Mosko, Phys. Rev. B 83, 245328 (2011); 84, 085454 (2011); K. Wakabayashi, Phys. Rev. B 64, 125428 (2001); A. Betti, G. Fiori, and G. Iannaccone, Phys. Rev. B 81, 035329 (2010); D. J. Mason, D. Prendergast, J. B. Neaton and E. J. Heller, Phys. Rev. B 84, 155401 (2011); P. A. Khomyakov and G. Brocks, Phys. Rev. B 70, 195402 (2004); P. A. Khomyakov, G. Brocks, V. Karpan, M. Zwierzycki, and P. J. Kelly, Phys. Rev. B 72, 035450 (2005); S. Tsukamoto, Y. Egami, K. Hirose and S. Blugel, Phys. Rev. B 84, 115443 (2011).

22 T. Ando, Phys. Rev. B 44,8017 (1991); K. Nikolic and A. MacKinnon, ibid. 50 , 11008 (1994).

${ }^{23}$ R. Tamura and M. Tsukada, Phys. Rev. B 55,4991 (1997) ; 61, 8548 (2000).

${ }^{24}$ Y. Meir and N. S. Wingreen, Phys. Rev. Lett. 68, 2512 (1992); T. Yamamoto, K. Watanabe and S. Watanabe, ibid. 95, 065501 (2005); H. Ness and A. J. Fisher, ibid. 83, 452 (1999); Y. Asai, ibid.93, 246102 (2004); T. Frederiksen, M. Brandbyge, N. Lorente and A.-P. Jauho, ibid.93, 256601 (2004); M. Paulsson,T. Frederiksen and M. Brandbyge, Phys. Rev. B 72, R201101 (2005); J.-X. Zhu and A. V. Balatsky, ibid. 67, 165326 (2003); H. Ness and L. K. Dash, ibid. 84, 235428 (2011); H. Ness, L. K. Dash and R. W. Godby, ibid. B 82, 085426 (2010);

${ }^{25}$ K. Hirose, N. Kobayashi and M. Tsukada, Phys. Rev. B 69, 245412 (2004); M. Brandbyge, J.-L. Mozos, P. Ordejon, J. Taylor and K. Stokbro, Phys. Rev. B 65, 165401 (2002).

${ }^{26}$ S. Datta, Electronic Transport in Mesoscopic Systems (Cambridge University Press, Cambridge 1995).

27 M. Buttiker, Y. Imry, R. Landauer and S. Pinhas, Phys. Rev. B 316207 (1985).

${ }^{28} \mathrm{Ph}$. Lambin, V. Meunier, and A. Rubio, Phys. Rev. B 62, 5129 (2000); Y.-K. Kwon and D. Tománek, Phys. Rev. B 58, R16001 (1998); Y. Miyamoto, S. Saito, and D. Tománek, ibid. 65, 041402 (2001).

29 J. T. Edwards and D. J. Thouless, J. Phys. C 5, 807 (1972). 